\title{
External Validation Study of First Trimester Obstetric Prediction Models (Expect Study I): Research Protocol and Population Characteristics
}

Linda Jacqueline Elisabeth Meertens ${ }^{1}$, MD, MSc; Hubertina CJ Scheepers ${ }^{2}$, MD, PhD; Raymond G De Vries ${ }^{3,4,5}$, $\mathrm{PhD}$; Carmen D Dirksen ${ }^{6}$, PhD; Irene Korstjens ${ }^{5}$, PhD; Antonius LM Mulder ${ }^{7}, \mathrm{MD}, \mathrm{PhD}$; Marianne J Nieuwenhuijze ${ }^{5}$, RM, PhD; Jan G Nijhuis ${ }^{2}$, MD, PhD; Marc EA Spaanderman ${ }^{2}$, MD, PhD; Luc JM Smits ${ }^{1}$, PhD

${ }^{1}$ Care and Public Health Research Institute (CAPHRI), Department of Epidemiology, Maastricht University, Maastricht, Netherlands

${ }^{2}$ School for Oncology and Developmental Biology (GROW), Department of Obstetrics and Gynecology, Maastricht University Medical Centre, Maastricht, Netherlands

${ }^{3}$ Center for Bioethics and Social Sciences in Medicine, University of Michigan Medical School, Ann Arbor, MI, United States

${ }^{4}$ Care and Public Health Research Institute (CAPHRI), Maastricht University, Maastricht, Netherlands

${ }^{5}$ Research Centre for Midwifery Science, Faculty of Health, Zuyd University, Maastricht, Netherlands

${ }^{6}$ Care and Public Health Research Institute (CAPHRI), Department of Clinical Epidemiology and Medical Technology Assessment (KEMTA), Maastricht University Medical Centre, Maastricht, Netherlands

${ }^{7}$ School for Oncology and Developmental Biology (GROW), Department of Pediatrics, Maastricht University Medical Centre, Maastricht, Netherlands

\section{Corresponding Author:}

Linda Jacqueline Elisabeth Meertens, MD, MSc

Care and Public Health Research Institute (CAPHRI)

Department of Epidemiology

Maastricht University

PO Box 616

Maastricht, $6200 \mathrm{MD}$

Netherlands

Phone: 31433882375

Fax: 31433884128

Email: linda.meertens@maastrichtuniversity.nl

\section{Abstract}

Background: A number of first-trimester prediction models addressing important obstetric outcomes have been published. However, most models have not been externally validated. External validation is essential before implementing a prediction model in clinical practice.

Objective: The objective of this paper is to describe the design of a study to externally validate existing first trimester obstetric prediction models, based upon maternal characteristics and standard measurements (eg, blood pressure), for the risk of pre-eclampsia (PE), gestational diabetes mellitus (GDM), spontaneous preterm birth (PTB), small-for-gestational-age (SGA) infants, and large-for-gestational-age (LGA) infants among Dutch pregnant women (Expect Study I). The results of a pilot study on the feasibility and acceptability of the recruitment process and the comprehensibility of the Pregnancy Questionnaire 1 are also reported.

Methods: A multicenter prospective cohort study was performed in The Netherlands between July 1, 2013 and December 31, 2015. First trimester obstetric prediction models were systematically selected from the literature. Predictor variables were measured by the Web-based Pregnancy Questionnaire 1 and pregnancy outcomes were established using the Postpartum Questionnaire 1 and medical records. Information about maternal health-related quality of life, costs, and satisfaction with Dutch obstetric care was collected from a subsample of women. A pilot study was carried out before the official start of inclusion. External validity of the models will be evaluated by assessing discrimination and calibration.

Results: Based on the pilot study, minor improvements were made to the recruitment process and online Pregnancy Questionnaire 1. The validation cohort consists of 2614 women. Data analysis of the external validation study is in progress. 
Conclusions: This study will offer insight into the generalizability of existing, non-invasive first trimester prediction models for various obstetric outcomes in a Dutch obstetric population. An impact study for the evaluation of the best obstetric prediction models in the Dutch setting with respect to their effect on clinical outcomes, costs, and quality of life-Expect Study II-is being planned.

Trial Registration: Netherlands Trial Registry (NTR): NTR4143; http://www.trialregister.nl/trialreg/admin/rctview.asp?TC=4143 (Archived by WebCite at http://www.webcitation.org/6t8ijtpd9)

(JMIR Res Protoc 2017;6(10):e203) doi: 10.2196/resprot.7837

\section{KEYWORDS}

external validation; first trimester; prediction models; pregnancy; risk assessment

\section{Introduction}

Perinatal mortality is an important quality indicator of perinatal care. The main causes of perinatal mortality are asphyxia, preterm birth (PTB), and born small-for-gestational-age (SGA) $[1,2]$. Pre-eclampsia (PE) is commonly related to SGA and induced preterm birth [3]. Another concern is the rising incidence of gestational diabetes mellitus (GDM), leading to large-for-gestational-age (LGA) infants [4]. Children born LGA are at increased risk of asphyxia and birth injuries [5]. Early identification of pregnancies at risk of these complications is important considering the substantial short- and long-term consequences for the health of mother and child. Women at high risk could benefit from further testing, increased surveillance, and preventive interventions.

A number of first trimester prediction models have been published addressing important obstetric outcomes including PE, GDM, spontaneous PTB, and infants born SGA or LGA [6]. These risk models are based on maternal characteristics, routine antenatal tests (eg, blood pressure), and sometimes include more complex predictors like specialized tests (eg, uterine artery Doppler and cervix length measurements) or biomarkers. Although some complex factors have been reported to improve discrimination, a drawback is that most of these tests provide additional costs, are not readily available in general antenatal settings, and are possibly inconvenient for pregnant women [7].

While the reported performance of most non-invasive prediction models is promising [7], few models have been externally validated in independent cohorts [8-16]. Evaluating the model's performance in another population than the one used for model development is crucial before applying a model in daily practice to guide patient care $[17,18]$.

This paper describes the design of a study aimed to externally validate existing first trimester obstetric prediction models, based upon maternal characteristics and standard measurements (eg, blood pressure), for the risk of PE, GDM, spontaneous PTB, and SGA and LGA infants among Dutch pregnant women (Expect Study I). Results of a pilot study on the feasibility and acceptability of the recruitment process and the comprehensibility of the Pregnancy Questionnaire 1 are also reported. Adequately performing models will be considered for use in clinical practice. We are planning an impact study-Expect Study II-to evaluate the application of adequately performing models (in association with tailored care paths) as compared with care-as-usual in Dutch obstetric care.

The specific objectives of the Expect Study I are (1) to identify published first trimester obstetric prediction models, based solely upon maternal characteristics and standard measurements (eg, blood pressure), for the outcomes PE, GDM, spontaneous PTB, SGA infants, and LGA infants; (2) to evaluate prospectively the predictive performance of these first trimester obstetric prediction models in a Dutch cohort of pregnant women; (3) to update, if necessary, the best performing models to the validation cohort; and (4) to measure maternal health-related quality of life, costs, and satisfaction aspects of current Dutch obstetric care for use as care-as-usual comparison to the intended Expect Study II.

\section{Methods}

\section{Selection of Prediction Models}

Systematic searches were performed in PubMed to identify all published first trimester obstetric prediction models using "prediction model" and its synonyms as search terms combined with relevant outcome terms and MeSH terms. The search terms were restricted to title and abstract fields (tiab). The detailed search strategies are available in Multimedia Appendix 1. Articles written in languages other than English, German, French, or Dutch were excluded. Citation lists of relevant articles were checked to select additional articles. The search was first performed in April 2013, before finalizing the study questionnaires, and will be updated before the start of each validation analysis per outcome. The first author screened all citations, and together with the last author, assessed the eligibility of the full text articles. In cases of disagreement, a third reviewer was used.

Prediction models were eligible for consideration if the following criteria were met: (1) the article presented the development of a prediction model or an update of a previously developed model, (2) the model contained multiple predictors, (3) predictors were routinely collected in Dutch obstetric care (maternal characteristics or blood pressure), (4) predictors were available and/or measured before 16 weeks and 0 days of gestation, (5) the model was based on weighted risk predictors, and (6) outcome of the model was PE, GDM, spontaneous PTB, SGA infants, or LGA infants. Authors of the original studies were contacted if the model intercept, regression coefficients, or definitions of predictors were not available. 


\section{Study Design and Population}

A multicentre prospective cohort study was performed among women living in the south-eastern part of The Netherlands (province of Limburg). Six hospitals and 36 midwifery practices recruited pregnant women less than 16 weeks pregnant and aged 18 years or older between July 1, 2013 and January 1, 2015. Follow-up took place until December 31, 2015. Pregnancies ending in a miscarriage, termination before 24 weeks of gestation, and women lost-to-follow-up were excluded.

The Medical Ethics Committee (MEC) of the Maastricht University Medical Centre evaluated the study protocol and declared that the study did not fall within the scope of the Dutch Medical Research Involving Human Subjects Act (WMO) (MEC 13-4-053). An independent physician was available for consultation by (eligible) participants.

\section{Recruitment}

Eligible pregnant women visiting their midwife (approximately $85 \%$ ) or obstetrician (approximately 15\%) in the first trimester of pregnancy received verbal and written information about Expect Study I [19]. They were also asked whether they were willing to receive further information by email or telephone. If so, contact details were entered into an online system by their caregiver and used to send an automated information email about the study. Pregnant women were asked to complete a Web-based questionnaire before 16 weeks of gestation (Pregnancy Questionnaire 1) and 6 weeks after the due date (Postpartum Questionnaire 1). During the visit, blood pressure and heart rate were routinely measured and the results were given in writing to the women on the information leaflet in order to self-report in Pregnancy Questionnaire 1 [20,21].

Study questionnaires could be accessed through the Expect Study website [22] by use of a personal login code contained in the written information and information email. Women agreeing to participate gave online informed consent and answered the eligibility criteria before the start of Pregnancy Questionnaire 1. Paper-and-pencil questionnaires were available upon request. Three reminders were sent by email during 3-day intervals if Pregnancy Questionnaire 1 was not accessed or incomplete. Women who completed Pregnancy Questionnaire 1 were invited 6 weeks after the due date to complete Postpartum Questionnaire 1. Three email reminders were sent during 6-day intervals, and in case of non-response, a paper-and-pencil version of Postpartum Questionnaire 1 was sent (provided that the postal address was available). In Pregnancy Questionnaire 1 , women were invited to fill out, on an optional basis, 3 additional questionnaires about costs, quality of life, and satisfaction of current obstetric care around 24 and 34 weeks of gestation (Pregnancy Questionnaires 2 and 3), and 6 weeks after the due date together with Postpartum Questionnaire 1 (Postpartum Questionnaire 2). Again, automatic reminders were sent out in case of non-response. Pregnancy status was asked at the beginning of Pregnancy Questionnaires 2 and 3. Women who reported a miscarriage or termination were referred to the end of the questionnaire and not invited for further questionnaires. Women not responding to Pregnancy Questionnaire 2 received no further invitations for the additional questionnaires, only for Postpartum Questionnaire 1. Women not responding to Pregnancy Questionnaire 3 were invited; however, for Postpartum Questionnaire 2. Medical records and discharge letters were requested from care providers.

A pilot study was carried out in the region of Maastricht before the official start of inclusion (March 25, 2013 to May 10, 2013) to assess the feasibility and acceptability of the recruitment process and the comprehensibility of Pregnancy Questionnaire 1. Evaluation questions about the recruitment process and form, content, and clarity of the questions were added to Pregnancy Questionnaire 1. If permission was given, participants were also approached by telephone.

\section{Data Collection}

Inclusion, follow-up, and data collection of participants were managed by use of a logistic application specifically developed for Expect Study I. Questionnaires were developed by the research team and where possible, validated questionnaires were included.

Pregnancy Questionnaire 1 contained questions about the following topics: socio-demographic characteristics, anthropometric data, medical conditions, obstetric history, lifestyle, medication, vitamin and mineral supplements, fruit intake, dietary intake of vitamin D and calcium (selection questions from the Dutch National Food Frequency Questionnaire tool [23]), sun exposure, family history of medical conditions and obstetric outcomes, mental health (Edinburgh Depression Scale [24,25]), health status (EQ-5D-3L and cognitive dimension [26,27]), current pregnancy, and blood pressure and heart rate measurements.

The following aspects were collected in Postpartum Questionnaire 1: pregnancy outcome, pregnancy complications, labor and delivery, and neonatal outcomes. We also added several questions about the biological father.

The additional questionnaires-Pregnancy Questionnaires 2 and 3 and Postpartum Questionnaire 2-assessed maternal health status (EQ-5D-3L and cognitive dimension [26,27]), state anxiety (State-Trait Anxiety Inventory [28]), patient satisfaction, and costs of current obstetric care. Satisfaction was assessed antepartum (Pregnancy Questionnaires 2 and 3) by means of the Patient Satisfaction Questionnaire Short Form (PSQ-18) [29] and postpartum (Postpartum Questionnaire 2 or delivered at Pregnancy Questionnaire 2 or 3) by the Pregnancy and Childbirth Questionnaire (PCQ) [30]. To evaluate the costs of current obstetric care, all midwifery, hospital, and other care institution costs associated with care for pregnant women and their newborns from the beginning of pregnancy up to around 6 weeks after the due date were requested. In Pregnancy Questionnaire 3 and Postpartum Questionnaire 2, the date of the last completed additional questionnaire was indicated so that participants could see what period was to be covered.

Data from the medical records and letters of discharge were extracted and entered into a predesigned datasheet using Microsoft Access. All records were verified by a second researcher. 
An overview of the items collected in the study questionnaires and data extracted from medical records and discharge letters is provided in Multimedia Appendix 2.

\section{Outcome Measures}

Primary study outcomes were maternal and perinatal adverse outcomes predicted by the selected prediction models. The maternal outcomes were PE and GDM. PE was defined as pregnancy induced hypertension (PIH) accompanied by proteinuria (at least $300 \mathrm{mg}$ protein in a 24 hour urine collection) [31]. PIH was defined as systolic blood pressure of at least 140 $\mathrm{mmHg}$ and/or diastolic blood pressure of at least $90 \mathrm{mmHg}$ (Korotkoff $\mathrm{V}$ ) after 20 weeks gestation, measured twice in a previously normotensive woman [31,32]. GDM was defined as a diagnosis of hyperglycemia during pregnancy, in a woman without pre-existing diabetes mellitus. The Dutch national guideline, in line with the World Health Organization guideline on Diagnosis and Classification of Diabetes Mellitus, defined hyperglycemia as the presence of either a fasting plasma glucose of $7.0 \mathrm{mmol} / \mathrm{l}$ or greater or 2-hour plasma glucose of $7.8 \mathrm{mmol} / \mathrm{l}$ or greater following a $75 \mathrm{~g}$ oral glucose tolerance test $[33,34]$. Perinatal outcomes included spontaneous PTB, SGA infants, and LGA infants. Spontaneous PTB is a delivery before 37 weeks of gestation started by primary contractions or spontaneous rupture of membranes. SGA and LGA were defined as an infant with a birth weight below the 10th percentile or above the 90th percentile, respectively, corrected for gestational age, ethnicity, gender, and parity [35].

The following secondary outcomes associated with the primary outcomes and important determinants of child morbidity and mortality were also measured: perinatal death (stillbirth or death within 7 days after birth, after 22 weeks of gestation), asphyxia (Apgar score of less than 7 after 5 minutes), admission to a neonatal intensive care unit (within 28 days after birth), SGA infants below the 2.3 percentile, PTB before 32 weeks of gestation, severe PE (delivery before the 34th completed week), instrumental delivery, cesarean section, and referral from midwife to obstetrician during delivery.

\section{Sample Size}

No generally accepted rules are available for the calculation of required sample sizes for external validation studies of prediction models. We followed the rule of thumb by Vergouwe et al (2005), which states that at least 100 events and 100 non-events are necessary in order to be able to detect relevant differences between model performance in the derivation set and the validation set [36]. Assuming that each primary outcome would affect $4 \%$ or more of the pregnancies, we needed to collect data from about 2500 women. We aimed to recruit 2750 women, allowing for $10 \%$ loss to-follow-up.

\section{Statistical Analysis}

Data analysis of the external validation study is in progress. Missing values will be handled by imputation, as analysis of only complete cases can lead to biased results [37]. Predictive performance of each prediction model will be evaluated by assessing discrimination and calibration [38,39]. Discrimination is the ability to distinguish between individuals who will develop the outcome from those who will not and will be assessed by calculating the c-index (area under the receiver operating characteristic curve [AUROC]). Calibration is the degree of agreement between predicted and observed probabilities. We will evaluate whether models may benefit from recalibration. Based on their final calibration and discriminative power, models will be ranked with respect to their predictive performance. The statistical analysis will be described in detail in the validation articles.

\section{Results}

\section{Pilot Study}

A total of 6 midwifery practices and 1 university hospital invited 95 pregnant women to participate. In total, $25(26 \%, 25 / 95)$ women gave informed consent, of whom $21(84 \%, 21 / 25)$ completed Pregnancy Questionnaire 1 fully and $4(16 \%, 4 / 25)$ only partially because of technical problems. Of the participants, $70(74 \%, 70 / 95)$ invited women who did not wish to fill out Pregnancy Questionnaire 1 could have return a non-participation form, but only 1 form was returned indicating that the woman "did not want to invest time in research." The participants were positive about the recruitment process and only minor revisions were needed in the content of Pregnancy Questionnaire 1. In reaction to the low response rate, we made improvements to the recruitment process by asking contact details of informed pregnant women to send reminders about the study by email or telephone. Furthermore, a leaflet was designed to make the written information more concise and attractive, and we distributed information through social media and posters for promotion. Lastly, half of the pilot study participants declared that an incentive would increase their motivation to participate, and that they preferred higher probability of receiving a low-cost reward in comparison to a lower chance of getting an expensive incentive. On the basis of this information, low-to-medium cost incentives were invoked in the recruitment procedure (lottery of 27 gift cards and 2 photo shoots).

\section{Validation Cohort}

The flowchart for enrolment and data collection of the validation cohort is shown in Figure 1. A total of 2794 women accessed the study website and gave online informed consent. Pregnancy Questionnaire 1 and Postpartum Questionnaire 1 were filled out by $2762(98.85 \%, 2762 / 2794)$ and $2178(78.86 \%$, 2178/2762) women, respectively. Medical records were retrieved for 2598 (94.06\%, 2598/2762) women. A completed Postpartum Questionnaire 1 or medical record was available for 2614 (94.64\%, 2614/2762) women (validation cohort). General baseline characteristics and the primary outcomes of the validation cohort are shown in Table 1. 
Table 1. Baseline characteristics and primary outcomes validation cohort of Expect Study I ( $\mathrm{N}=2614)$.

\begin{tabular}{cl}
\hline Baseline characteristics (less than 16 weeks of gestation) & Observed valida \\
\hline Age in years, mean (SD) & $30.2(3.9)$ \\
Ethnicity & \\
Caucasian & $2533(96.90 \%)$ \\
African-Caribbean & $3(0.11 \%)$ \\
Asian & $20(0.77 \%)$ \\
Hispanic & $11(0.42 \%)$ \\
Mixed & $47(1.80 \%)$ \\
Tertiary education & $1420(54.32 \%)$ \\
BMI ${ }^{\mathbf{a}}$, mean (SD) & $24.2(4.3)$ \\
$<18.5$ & $87(3.33 \%)$ \\
$18.5-24.9$ & $1665(63.70 \%)$ \\
$25-29.9$ & $585(22.38 \%)$ \\
$30-39.9$ & $263(10.06 \%)$ \\
$\geq 40$ & $9(0.34 \%)$
\end{tabular}

Medical history

Chronic hypertension

Diabetes mellitus

Renal disease

Smoking during pregnancy

Ever

Current

Alcohol consumption during pregnancy

Ever

Current

Nulliparous

\section{Conception}

Spontaneous

Ovulation induction

$\mathrm{IVF}^{\mathrm{b}} / \mathrm{ICSI}^{\mathrm{c}}$

\section{Obstetric history}

Prior pre-eclampsia

Prior gestational diabetes mellitus

Prior preterm birth $<37$ weeks of gestation

Prior birth weight $<10$ th percentile

Prior birth weight $>90$ th percentile

\section{Primary outcomes}

Pre-eclampsia

Gestational diabetes mellitus

Spontaneous PTB $<37$ weeks of gestation

Birth weight $<10$ th percentile

Birth weight $>90$ th percentile
$28(1.07 \%)$

$11(0.42 \%)$

$5(0.19 \%)$

$318(12.17 \%)$

$157(6.01 \%)$

$479(18.32 \%)$

$9(0.34 \%)$

$1326(50.73 \%)$

$2440(93.34 \%)$

$93(3.56 \%)$

$81(3.10 \%)$

$72(2.75 \%)$

$15(0.57 \%)$

$141(5.39 \%)$

$108(4.13 \%)$

$170(6.50 \%)$

$76(2.91 \%)$

$74(2.83 \%)$

$127(4.86 \%)$

$206(7.88 \%)$

$224(8.57 \%)$ 


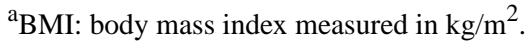

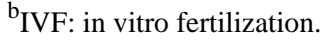

${ }^{\mathrm{c}}$ ICSI: intracytoplasmic sperm injection.

Of the women included in the validation cohort, 1548 (59.22\%, $1548 / 2614$ ) gave permission to be invited for the additional questionnaires. Pregnancy Questionnaire 2 was filled out by $891(57.56 \%, 891 / 1548)$ women. Of the women who started the first additional Pregnancy Questionnaire 2 and were still pregnant, 795 (89.5\%, 795/888) women filled out Pregnancy Questionnaire 3. Postpartum Questionnaire 2 was filled out by $744(83.5 \%, 744 / 891)$ women.

Figure 1. Inclusion and data collection of Expect Study I. The components in the dotted box represent the additional questionnaires. A total of 1548 participants gave permission to receive additional questionnaires (a).

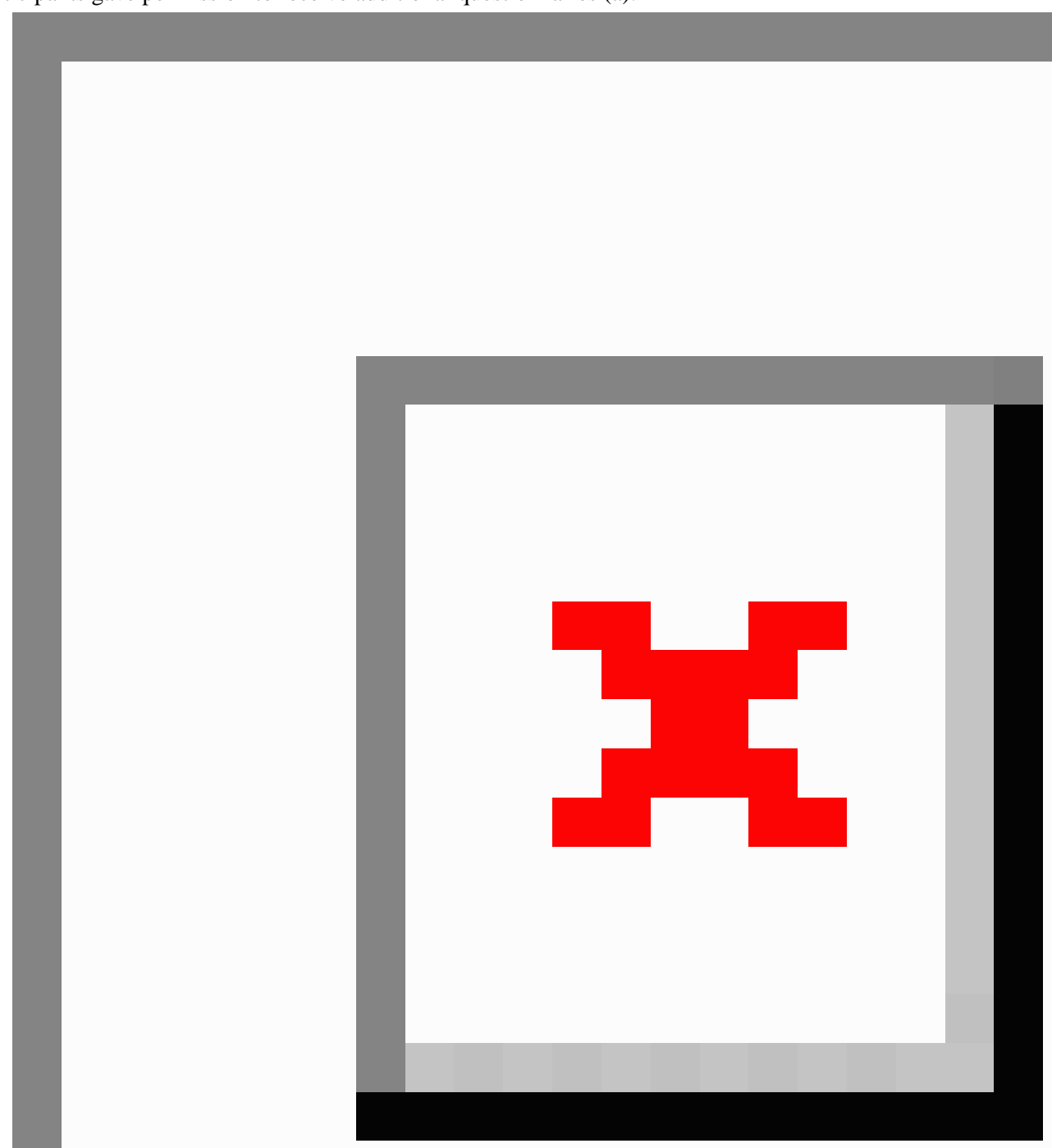

\section{Discussion}

Here, we describe the protocol of a study that aims to assess the predictive performance of multiple first trimester obstetric prediction models within an independent Dutch population. In this way, prediction models with similar outcomes can be compared and best performing models can be selected [40].

In the evaluation of a prediction model, external validation is an essential step. Generally, the predictive performance of the 
model decreases in the validation dataset due to model over-fitting in the development cohort [18,41]. Existing independent external validation studies of non-invasive, first trimester obstetric prediction models for GDM showed stable discriminative performances, with the highest AUROCs for the models by Nanda et al (AUROC 0.79) and Van Leeuwen et al (AUROC 0.76-0.77) [13-16]. For the outcomes early and late PE, only a few models based upon maternal characteristics and blood pressure have been externally validated and AUROCs declined to around 0.70 [8-12]. A limitation is that the numbers of events in these validation studies were (extremely) low, especially for early PE. No independent external validation studies of non-invasive prediction models for overall PE, spontaneous PTB, SGA infants, and LGA infants have been published.

The main strength of our study is the prospective cohort design, which enables optimal measurement of predictors and outcomes [42]. Recruitment by multiple centers improves the likelihood of obtaining a representative sample of the obstetric population, which is especially important in the obstetric care system in The Netherlands in which most pregnant women start antenatal care with a midwife. Web-based questionnaires were used as a data collection tool, which is efficient in a population with high access to the Internet, as it improves data quality and less missing data due to the incorporation of validation checks. Moreover, it is also more user-friendly in comparison to paper-pencil forms as non-relevant follow-up questions could be hidden, speeding up completion [43].

If one or more prediction models turns out to be externally valid, eventually after model updating, it is not self-evident that the model will be useful in clinical practice. The prediction models can only lead to improved outcomes for mother and child if they can guide healthcare professionals and individuals in their decision making regarding further management that are tailored to individual risk profiles, including additional testing, preventive interventions, lifestyle changes, monitoring, or treatment [42].

Statistical performance measures are important aspects of a prediction model, but they do not indicate its clinical usefulness. Even if the statistical performance is less good, the model may predict better compared to usual practice, and vice versa [44-46]. We plan to evaluate the clinical utility of the validated models by decision analysis. Decision analysis provides insight whether the model is better than usual care by combining test characteristics with evidence on consequences of the outcome, effects and burden of the further management, and costs [18]. In case a model is worth considering for implementation in clinical practice, it is necessary to determine the most optimal threshold value for risk classification. Finally, we will assess the effects of applying prediction models with tailored care paths on decision-making and health outcomes in Dutch obstetric care, as compared with care-as-usual (Expect Study II).

\section{Acknowledgments}

The Expect Study is independently funded by The Netherlands Organization for Health Research and Development, Pregnancy and Childbirth Program (ZonMw grant 209020007).

We gratefully acknowledge all of the women who participated in the pilot study and prospective cohort study I. Expect Study I could not have been established without the contribution of the following participating midwifery practices and departments of obstetrics and gynecology of hospitals in the Province of Limburg: Zuyderland Medical Center Heerlen and Sittard-Geleen, Maastricht University Medical Center, Laurentius Hospital Roermond, St. Jans Gasthuis Weert, VieCuri Medical Center, midwifery practice Roermond, midwifery practice Nederweert, midwifery practice Weert, midwifery practice Lenie \& Chantal, midwifery practice Loes Wijnhoven, midwifery practice De Roerstreek, midwifery practice Bollebuik, midwifery practice Westenberg, midwifery practice Cranendonck, midwifery practice Becca, midwifery practice Born, midwifery practice Geleen, midwifery practice Grevenbicht, midwifery practice Sittard, midwifery practice Sittard-Oost, midwifery practice Stein, midwifery practice Astrea, midwifery practice Horst \& Maasdorpen, midwifery practice Reuver-Tegelen, midwifery practice Janneke van Hal, midwifery practice Raijer \& Sup, midwifery practice Venlo-Blerick, midwifery practice Venray, midwifery practice Schoffelen-Van Vleuten, midwifery practice Maastricht, midwifery practice Meerssen, midwifery practice Naomi Satijn, midwifery practice Vita, midwifery practice Het Verloskundig Huis, midwifery practice Valkenburg, midwifery practice Parkstad, midwifery practice Lief, midwifery practice Bevalt Beter, midwifery practice 't Bolleke, midwifery practice Natuurlijk bij Jeanny, and midwifery practice La Vie. We also acknowledge GroenekruisDomicura, Cicogna, ZiNkraamzorg, and maternity center Echt for distributing study information leaflets. Lastly, we thank all research assistants that were involved in this study and Memic (Center for Data and Information Management) for developing and maintaining the logistic system and data management.

\section{Authors' Contributions}

The study was developed by LS, HS, and MS in collaboration with RDV, CD, IK, AM, MN, and JN. All collaborators are considered as co-authors as they have significantly contributed to the design of the study. LM elaborated and carried out Expect Study I under the supervision of LS and HS. The manuscript was drafted by LM in collaboration with LS and HS. All authors critically reviewed and approved the final manuscript.

\section{Conflicts of Interest}

None declared 


\section{Multimedia Appendix 1}

Search strategies first-trimester obstetric prediction models.

[PDF File (Adobe PDF File), 5KB-Multimedia Appendix 1]

\section{Multimedia Appendix 2}

Data collection study questionnaires and medical records.

[PDF File (Adobe PDF File), 610KB-Multimedia Appendix 2]

\section{Multimedia Appendix 3}

Reviewer comments (3 reviewers) and rebuttal grant application ZonMw. The Netherlands Organization for Health Research and Development, Pregnancy and Childbirth program (ZonMw grant 209020007). [ENGLISH].

[PDF File (Adobe PDF File), 1MB-Multimedia Appendix 3]

\section{References}

1. Bonsel GJ, Birnie E, Denktas S, Poeran J, Steegers EAP. Lijnen in de Perinatale Sterfte, Signalementstudie Zwangerschap en Geboorte 2010. Rotterdam: Erasmus MC; 2010. URL: https://repub.eur.nl/pub/23454 [accessed 2017-03-28] [WebCite Cache ID 6tCnqTpQ3]

2. van der Kooy J, Poeran J, de Graaf JP, Birnie E, Denktasş S, Steegers EA, et al. Planned home compared with planned hospital births in the Netherlands: intrapartum and early neonatal death in low-risk pregnancies. Obstet Gynecol 2011 Nov;118(5):1037-1046. [doi: 10.1097/AOG.0b013e3182319737] [Medline: 22015871]

3. Mol BWJ, Roberts CT, Thangaratinam S, Magee LA, de Groot CJM, Hofmeyr GJ. Pre-eclampsia. Lancet 2016 Mar 05;387(10022):999-1011. [doi: 10.1016/S0140-6736(15)00070-7] [Medline: 26342729]

4. Koning SH, Hoogenberg K, Lutgers HL, van den Berg PP, Wolffenbuttel BH. Gestational diabetes mellitus:current knowledge and unmet needs. J Diabetes 2016 Nov;8(6):770-781. [doi: 10.1111/1753-0407.12422] [Medline: 27121958]

5. Araujo Júnior E, Peixoto AB, Zamarian ACP, Elito Júnior J, Tonni G. Macrosomia. Best Pract Res Clin Obstet Gynaecol 2017 Jan;38:83-96. [doi: 10.1016/j.bpobgyn.2016.08.003] [Medline: 27727018]

6. Kleinrouweler CE, Cheong-See FM, Collins GS, Kwee A, Thangaratinam S, Khan KS, et al. Prognostic models in obstetrics: available, but far from applicable. Am J Obstet Gynecol 2016 Jan;214(1):79-90.e36. [doi: 10.1016/j.ajog.2015.06.013] [Medline: 26070707]

7. Al-Rubaie Z, Askie LM, Ray JG, Hudson HM, Lord SJ. The performance of risk prediction models for pre-eclampsia using routinely collected maternal characteristics and comparison with models that include specialised tests and with clinical guideline decision rules: a systematic review. BJOG 2016 Aug;123(9):1441-1452 [FREE Full text] [doi: 10.1111/1471-0528.14029] [Medline: 27225348]

8. Herraiz I, Arbués J, Camaño I, Gómez-Montes E, Grañeras A, Galindo A. Application of a first-trimester prediction model for pre-eclampsia based on uterine arteries and maternal history in high-risk pregnancies. Prenat Diagn 2009 Dec;29(12):1123-1129. [doi: 10.1002/pd.2383] [Medline: 19813221]

9. Farina A, Rapacchia G, Freni Sterrantino A, Pula G, Morano D, Rizzo N. Prospective evaluation of ultrasound and biochemical-based multivariable models for the prediction of late pre-eclampsia. Prenat Diagn 2011 Dec;31(12):1147-1152. [doi: 10.1002/pd.2849] [Medline: 22009522]

10. Park FJ, Leung CH, Poon LC, Williams PF, Rothwell SJ, Hyett JA. Clinical evaluation of a first trimester algorithm predicting the risk of hypertensive disease of pregnancy. Aust N Z J Obstet Gynaecol 2013 Dec;53(6):532-539. [doi: 10.1111/ajo.12126] [Medline: 23919594]

11. Skråstad RB, Hov GG, Blaas HK, Romundstad PR, Salvesen KÅ. Risk assessment for preeclampsia in nulliparous women at 11-13 weeks gestational age: prospective evaluation of two algorithms. BJOG 2015 Dec;122(13):1781-1788 [FREE Full text] [doi: 10.1111/1471-0528.13194] [Medline: 25471057]

12. E Holanda Moura SB, Park F, Murthi P, Martins WP, Kane SC, Williams P, et al. TNF-R1 as a first trimester marker for prediction of pre-eclampsia. J Matern Fetal Neonatal Med 2016 Mar;29(6):897-903. [doi: 10.3109/14767058.2015.1022865] [Medline: 25758630]

13. van Leeuwen M, Opmeer BC, Zweers EJK, van Ballegooie E, ter Brugge HG, de Valk HW, et al. External validation of a clinical scoring system for the risk of gestational diabetes mellitus. Diabetes Res Clin Pract 2009 Jul;85(1):96-101. [doi: 10.1016/j.diabres.2009.04.025] [Medline: 19477547]

14. Thériault S, Forest J, Massé J, Giguère Y. Validation of early risk-prediction models for gestational diabetes based on clinical characteristics. Diabetes Res Clin Pract 2014 Mar;103(3):419-425. [doi: 10.1016/j.diabres.2013.12.009] [Medline: 24447804] 
15. Syngelaki A, Pastides A, Kotecha R, Wright A, Akolekar R, Nicolaides KH. First-trimester screening for gestational diabetes mellitus based on maternal characteristics and history. Fetal Diagn Ther 2015;38(1):14-21 [FREE Full text] [doi: $\underline{10.1159 / 000369970]}$ [Medline: 25531073]

16. Lamain-de Ruiter M, Kwee A, Naaktgeboren CA, de Groot I, Evers IM, Groenendaal F, et al. External validation of prognostic models to predict risk of gestational diabetes mellitus in one Dutch cohort: prospective multicentre cohort study. BMJ 2016 Aug 30;354:i4338 [FREE Full text] [Medline: 27576867]

17. Justice AC, Covinsky KE, Berlin JA. Assessing the generalizability of prognostic information. Ann Intern Med 1999 Mar 16;130(6):515-524. [Medline: 10075620]

18. Steyerberg E. Clinical Prediction Models a Practical Approach to Development, Validation, and Updating. New York: Springer Science; 2009.

19. Perined. Perinatale Zorg in Nederland 2014. Utrecht: Perined; 2015. URL: https://assets.perined.nl/docs/ 353d9249-9875-4cb3-9c86-f078ae3f7aef.pdf [accessed 2017-03-28] [WebCite Cache ID 6pIi8Urgh]

20. Nederlandse Vereniging voor Obstetrie en Gynaecologie. Addendum bij multidisciplinaire richtlijn Hypertensieve aandoeningen in de zwangerschap uit 2011. 2014. URL: http://www.nvog-documenten.nl/uploaded/docs/

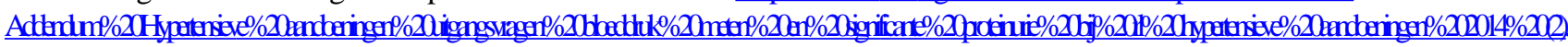
pdf [accessed 2017-03-28] [WebCite Cache ID 6pIQB9yiH]

21. Koninklijke Nederlandse Organisatie van Verloskundigen. Standaard Hypertensieve aandoeningen tijdens de zwangerschap, bevalling en kraamperiode. 2011. URL: http://www.knov.nl/fms/file/knov.nl/knov downloads/393/file/ KNOV_Standaard_Hypertensie_versie2012.pdf?download_category=richtlijnen-praktijkkaarten [accessed 2017-03-28] [WebCite Cache ID 6pIR7aesd]

22. Expect Studie. Wetenschappelijk onderzoek bij zwangere vrouwen in de provincie Limburg URL: https://www. zwangerinlimburg.nl/ [accessed 2017-09-01] [WebCite Cache ID 6t8nQZrB6]

23. Molag ML, de Vries JH, Duif N, Ocké MC, Dagnelie PC, Goldbohm RA, et al. Selecting informative food items for compiling food-frequency questionnaires: comparison of procedures. Br J Nutr 2010 Aug;104(3):446-456. [doi: 10.1017/S0007114510000401] [Medline: 20374682]

24. Bergink V, Kooistra L, Lambregtse-van den Berg MP, Wijnen H, Bunevicius R, van Baar A, et al. Validation of the Edinburgh Depression Scale during pregnancy. J Psychosom Res 2011 Apr;70(4):385-389. [doi:

10.1016/j.jpsychores.2010.07.008] [Medline: 21414460]

25. Bunevicius A, Kusminskas L, Pop VJ, Pedersen CA, Bunevicius R. Screening for antenatal depression with the Edinburgh Depression Scale. J Psychosom Obstet Gynaecol 2009 Dec;30(4):238-243. [doi: 10.3109/01674820903230708] [Medline: 19845492]

26. EuroQol Group. EuroQol--a new facility for the measurement of health-related quality of life. Health Policy 1990 Dec;16(3):199-208. [Medline: 10109801]

27. Krabbe PF, Stouthard ME, Essink-Bot ML, Bonsel GJ. The effect of adding a cognitive dimension to the EuroQol multiattribute health-status classification system. J Clin Epidemiol 1999 Apr;52(4):293-301. [Medline: 10235169]

28. van der Ploeg HM, Defares P, Spielberger CD. Handleiding bij de Zelf-beoordelings Vragenlijst ZBV. Een nederlandstalige bewerking van de Spielberger State-Trait Anxiety Inventory. Lisse: Swets \& Zeitlinger; 1980.

29. Marshall GN, Hays RD. The patient satisfaction questionnaire short-form (PSQ-18). Santa Monica, CA: RAND Corporation; 1994. URL: https://www.rand.org/content/dam/rand/pubs/papers/2006/P7865.pdf[WebCite Cache ID 6trkWz2FZ]

30. Truijens SE, Pommer AM, van Runnard Heimel PJ, Verhoeven CJ, Oei SG, Pop VJ. Development of the Pregnancy and Childbirth Questionnaire (PCQ): evaluating quality of care as perceived by women who recently gave birth. Eur J Obstet Gynecol Reprod Biol 2014 Mar;174:35-40. [doi: 10.1016/j.ejogrb.2013.11.019] [Medline: 24332094]

31. Nederlandse Vereniging voor Obstetrie en Gynaecologie. Richtlijn Hypertensieve aandoeningen in de zwangerschap. 2005. URL: http://nvog-documenten.nl/uploaded/docs/Hypertensieve\%20aandoeningen\%20in\%20de\%20zwangerschap.pdf [accessed 2017-03-28] [WebCite Cache ID 6pISdcuWd]

32. Brown MA, Hague WM, Higgins J, Lowe S, McCowan L, Oats J, Austalasian Society of the Study of Hypertension in Pregnancy. The detection, investigation and management of hypertension in pregnancy: full consensus statement. Aust $\mathrm{N}$ Z J Obstet Gynaecol 2000 May;40(2):139-155. [Medline: 10925900]

33. World Health Organization. Definition, Diagnosis and Classification of Diabetes Mellitus and its Complications. Report of a WHO Consultation. Geneva: World Health Organization; 1999. Part 1: diagnosis and classification of diabetes mellitus URL: http://apps.who.int/iris/bitstream/10665/66040/1/WHO NCD NCS 99.2.pdf [accessed 2017-03-28] [WebCite Cache ID 6pIjZsyY5]

34. Nederlandse Vereniging voor Obstetrie en Gynaecologie. Richtlijn Diabetes Mellitus en Zwangerschap (2.0). 2010. URL: http://nvog-documenten.nl/richtlijn/doc/index.php?type=save\&richtlijn id=863 [accessed 2017-03-28] [WebCite Cache ID 6pISi71FQ]

35. Visser GHA, Eilers PHC, Elferink-Stinkens PM, Merkus HM, Wit JM. New Dutch reference curves for birthweight by gestational age. Early Hum Dev 2009 Dec;85(12):737-744. [doi: 10.1016/j.earlhumdev.2009.09.008] [Medline: 19914013] 
36. Vergouwe Y, Steyerberg EW, Eijkemans MJ, Habbema JD. Substantial effective sample sizes were required for external validation studies of predictive logistic regression models. J Clin Epidemiol 2005 May;58(5):475-483. [doi: 10.1016/j.jclinepi.2004.06.017] [Medline: 15845334 ]

37. van der Heijden GJ, Donders AR, Stijnen T, Moons KG. Imputation of missing values is superior to complete case analysis and the missing-indicator method in multivariable diagnostic research: a clinical example. J Clin Epidemiol 2006 Oct;59(10):1102-1109. [doi: 10.1016/j.jclinepi.2006.01.015] [Medline: 16980151]

38. Steyerberg EW, Vergouwe Y. Towards better clinical prediction models: seven steps for development and an ABCD for validation. Eur Heart J 2014 Aug 01;35(29):1925-1931 [FREE Full text] [doi: 10.1093/eurheartj/ehu207] [Medline: 24898551]

39. Debray TP, Vergouwe Y, Koffijberg H, Nieboer D, Steyerberg EW, Moons KG. A new framework to enhance the interpretation of external validation studies of clinical prediction models. J Clin Epidemiol 2015 Mar;68(3):279-289 [FREE Full text] [doi: 10.1016/j.jclinepi.2014.06.018] [Medline: 25179855]

40. Collins GS, Moons KG. Comparing risk prediction models. BMJ 2012 May 24;344:e3186. [Medline: 22628131]

41. Moons KG, Kengne AP, Grobbee DE, Royston P, Vergouwe Y, Altman DG, et al. Risk prediction models: II. External validation, model updating, and impact assessment. Heart 2012 May;98(9):691-698. [doi: 10.1136/heartjnl-2011-301247] [Medline: 22397946]

42. Moons KG, Royston P, Vergouwe Y, Grobbee DE, Altman DG. Prognosis and prognostic research: what, why, and how? BMJ 2009 Feb 23;338:b375. [Medline: 19237405]

43. van Gelder MM, Bretveld RW, Roeleveld N. Web-based questionnaires: the future in epidemiology? Am J Epidemiol 2010 Dec 01;172(11):1292-1298. [doi: 10.1093/aje/kwq291] [Medline: 20880962]

44. Altman DG, Royston P. What do we mean by validating a prognostic model? Stat Med 2000 Feb 29;19(4):453-473. [Medline: 10694730]

45. Moons KG, Altman DG, Vergouwe Y, Royston P. Prognosis and prognostic research: application and impact of prognostic models in clinical practice. BMJ 2009 Jun 04;338:b606. [Medline: 19502216]

46. Altman DG, Vergouwe Y, Royston P, Moons KG. Prognosis and prognostic research: validating a prognostic model. BMJ 2009 May 28;338:b605. [Medline: 19477892]

\author{
Abbreviations \\ AUROC: area under the receiver operating characteristic \\ GDM: gestational diabetes mellitus \\ LGA: large-for-gestational-age \\ MEC: medical ethical committee \\ PE: pre-eclampsia \\ PIH: pregnancy induced hypertension \\ PTB: preterm birth \\ SGA: small-for-gestational-age
}

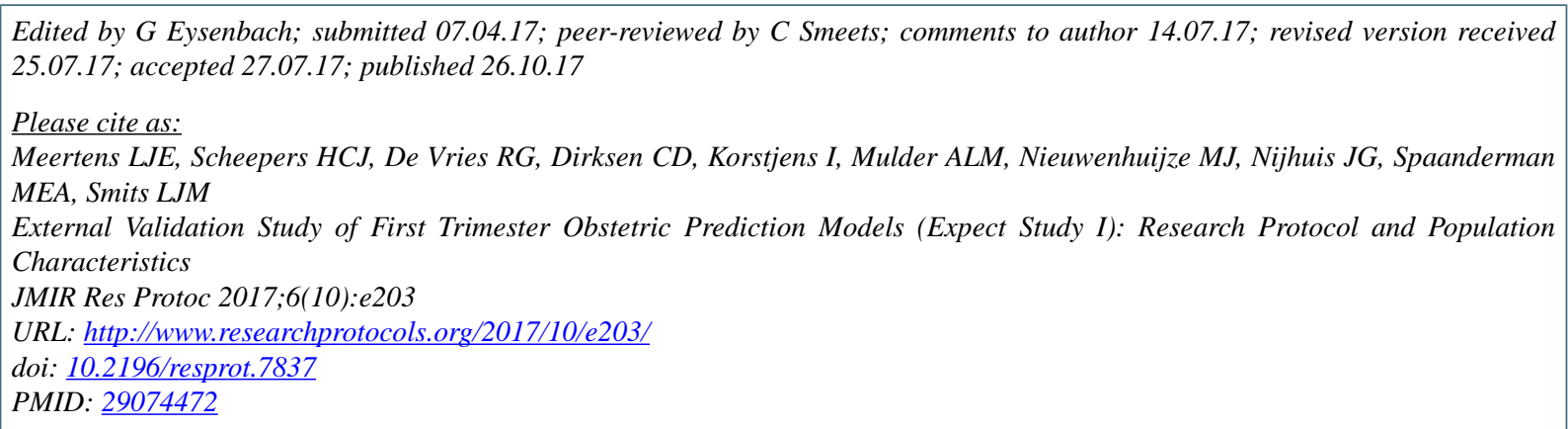

CLinda Jacqueline Elisabeth Meertens, Hubertina CJ Scheepers, Raymond G De Vries, Carmen D Dirksen, Irene Korstjens, Antonius LM Mulder, Marianne J Nieuwenhuijze, Jan G Nijhuis, Marc EA Spaanderman, Luc JM Smits. Originally published in JMIR Research Protocols (http://www.researchprotocols.org), 26.10.2017. This is an open-access article distributed under the terms of the Creative Commons Attribution License (https://creativecommons.org/licenses/by/4.0/), which permits unrestricted use, distribution, and reproduction in any medium, provided the original work, first published in JMIR Research Protocols, is 
properly cited. The complete bibliographic information, a link to the original publication on http://www.researchprotocols.org, as well as this copyright and license information must be included. 